\title{
Hemorrhagic extrahepatic recurrence of hepatocellular carcinoma: A case report
}

\author{
Gilbert Peh Voon Yeow ${ }^{1}$, David Gan Eng Yeow ${ }^{1}$, Padmaan Sankaran ${ }^{1}$, \\ Thanesh Maiyauen ${ }^{1}$, and Mohd Sharifudin Sharif ${ }^{2}$
}

\author{
${ }^{1}$ General Surgery Department, Hospital Queen Elizabeth, ${ }^{2}$ Gleneagles Kota Kinabalu, Sabah, Malaysia
}

\begin{abstract}
The recurrence of hepatocellular carcinoma $(\mathrm{HCC})$ can mainly be divided into two phases or types: the intrahepatic recurrence (IHR) and the extrahepatic recurrence (EHR). Generally speaking, the EHR is usually referenced as being the less common of the two types or categories. In a post-hemihepatectomy patient with routine surveillance at 6 months, it was noted that the patient appeared to have an elevated alpha-fetoprotein, and also complained of vague lower abdominal pain. The accompanying computed tomography scan revealed a $6 \mathrm{~cm}$-sized heterogeneous mass located at the pelvis region. Next, during a laparotomy for resection, the tumor was found to be bleeding from the omental deposition. In conclusion, a high index of suspicion and early surgical intervention can help in detecting possible bleeding extrahepatic recurrence in the background of a patient with history of HCC. (Ann Hepatobiliary Pancreat Surg 2019;23:77-79)
\end{abstract}

Key Words: Hepatocellular carcinoma; Omental recurrence; Recurrence; Extrahepatic

\section{INTRODUCTION}

Hepatocellular carcinoma (HCC) is a common health problem worldwide. It usually parallels the prevalence of a viral hepatitis. In what follows with development of advanced surgical techniques and technology, the option of a resection procedure has remained the first line of treatment to achieve a cure for HCC in patients with satisfactory liver function reserve and who are the status of being satisfactory anatomically resectable candidates for the procedure. ${ }^{1,2}$ However the prognosis of post-hepatectomy patients still remains unsatisfactory with a documented recurrence of $50-80 \%$ at 5 years. ${ }^{1}$ The recurrence can generally be divided in to intrahepatic recurrence (IHR) only and extrahepatic recurrence (EHR) cases or patient groups. In this sense, the EHR is less common than IHR; $16 \%$, and patients with EHR have a much poorer prognosis. ${ }^{3}$ This is probably as a result of the fact that these tumors are usually multiple, aggressive and therefore not always amenable to the use of a curative re- section, and there are no established systemic therapies to recommend for an EHR of HCC.

\section{CASE}

We herein present a patient with a bleeding EHR occurring within 1 year after curative right hemihepatectomy. A 51-year-old female, with a background history of hepatitis $\mathrm{B}$ carrier for which she is on oral tenofovir, presented with a 5-month history of dull right hypochondriac pain. On examination, no abdominal mass was found at that time. Additionally, the blood investigation results revealed an alpha-fetoprotein (AFP) of $8334 \mathrm{ng} / \mathrm{ml}$. A multiphase computed tomography (CT) of the liver showed a segment V \& VI mass with peripheral enhancement, with a much smaller satellite nodule in the segment 6 , for which the patient underwent a right hemihepatectomy on 7th April 2016. Besides superficial surgical site infection, the patient recovered well and was discharged on postsurgery day 13. Subsequently, the histopathological ex-

Received: May 3, 2018; Revised: July 11, 2018; Accepted: July 13, 2018

Corresponding author: Gilbert Peh Voon Yeow

General Surgery Department, Hospital Queen Elizabeth 1, 13A, Jalan Penampang, Kota Kinabalu, Sabah, 88200, Malaysia Tel: +6088-517555, Fax: +6088-21199, E-mail: gilbert_yeow@hotmail.com

Copyright (C) 2019 by The Korean Association of Hepato-Biliary-Pancreatic Surgery

This is an Open Access article distributed under the terms of the Creative Commons Attribution Non-Commercial License (http://creativecommons.org/ licenses/by-nc/4.0) which permits unrestricted non-commercial use, distribution, and reproduction in any medium, provided the original work is properly cited. Annals of Hepato-Biliary-Pancreatic Surgery • pISSN: 2508-5778 - elSSN: 2508-5859 
amination showed a moderately differentiated HCC Grade II, with the nearest resection margin measured at $1.5 \mathrm{~cm}$, as well as the focal capsular involvement, venous invasion and background of steatosis.

During follow-up, AFP reduced to $65 \mathrm{ng} / \mathrm{ml}$, and a multiphase CT liver showed no enhancing liver lesion. During the 6th month follow-up, it was noted that AFP was $3284 \mathrm{ng} / \mathrm{ml}$. A repeat CT multiphase on 5th December 2016 (8 months post-surgery) showed no enhancing liver lesions, but did note an irregular heterogeneous mass with characteristic surrounding fat streakiness in the pelvis thus measuring $6.6 \mathrm{~cm} \times 6.5 \mathrm{~cm} \times 6.5 \mathrm{~cm}$.

The patient was scheduled for a diagnostic laparoscopy with the intention of localizing and assessing the option of resectability. On the night before the surgery, the patient started experiencing vague abdominal pain (retrospective) for which the patient ignored. Her vital signs were recorded as stable overnight. The laparoscopy found that the the omentum was adhered to the pelvic region, and it was found that there were blood and clots located in the peritoneal cavity. An immediate decision was made to convert the surgery to a midline laparotomy. The finding was an omental mass measuring $10 \mathrm{~cm}$, which was loosely adhered to the dome of the bladder, which was freed easily with the use of a blunt dissection. The tumor was resected en-bloc with omentum. The patient recovered well and was discharged on post-surgery day 5 . The associated histopathology report showed a metastatic HCC. A repeat CT a month after the surgery showed no recurrence and AFP level dropped to $108.2 \mathrm{ng} / \mathrm{ml}$.

\section{DISCUSSION}

In patients with $\mathrm{HCC}$, it is noted that bleeding or presence of ruptured HCC is not a common occurrence. A single center in Hong Kong documented a 9\% incidence of bleeding HCC from the total number of cases of HCC seen over a 10 year period. ${ }^{3}$ The diagnosis may be difficult, as this condition may mimic other forms of intraabdominal pathologies, and requires a high index of suspicion. As of now there are no standard algorithms of related treatment approaches to be utilized in dealing with recurrent HCC. The definitive management usually ranges from conservative, the use of minimally invasive approaches via endovascular embolization to surgery with plication, hepatic artery ligation, alcohol injection, double stage procedure (packing then definitive) or definitive procedure in a single setting. ${ }^{4}$ In these cases, the 30 -day mortality was measured to be $38 \%{ }^{3}$

The pattern of extrahepatic recurrence can then further be grouped into 3 patterns. Pattern I represented patients whose first recurrence was noted in the liver and in whom an extrahepatic metastasis developed later after repetitive locoregional treatments; pattern II included patients in whom both intrahepatic and extrahepatic recurrences were evident when recurrence was identified; and pattern III included patients who had only extrahepatic metastasis without intrahepatic metastases at the time of diagnosis of metastasis. Out of these groups, it is noted that the group 3 is considered the rarest group. ${ }^{5}$

The incidence of bleeding in a metastatic HCC is uncommon. Not many recent case reports are available discussing the incidence of a ruptured bleeding metastatic/recurrent HCC. One case shows a post mortem report of a ruptured peri-pancreatic lymph node; along with a histopathological examination of which returned as conclusively reported metastatic HCC. ${ }^{6}$ Another article discussed two patients being treated with HCC who were originally presenting with an acute abdomen. ${ }^{7}$ In these cases, with the CT imaging showing peritoneal tumor seeding and hematoma, the patients were operated to achieve hemostasis. The nodules which were sent for histopathological examination also confirmed the presence of a metastatic HCC. $^{7}$

These bring us to understand that the route of extrahepatic HCC spread has been postulated from travel through tumor cells via lymphatics, vessels, direct extension or seedling. ${ }^{8}$ In this case report, the possibility of existing tumor deposit during the first operation cannot be ruled out, although the serum AFP did show a drastic drop post-surgery.

In conclusion, a bleeding metastatic HCC can be equally difficult to diagnose and as difficult to treat. A high index of suspicion is required in these cases, and an intervention should be recommended after a full review as on a timely basis. There are no protocols for this type of treatment, therefore treatment should be tailored to each patient and patient situation. 


\section{REFERENCES}

1. Omata M, Lesmana LA, Tateishi R, Chen PJ, Lin SM, Yoshida $\mathrm{H}$, et al. Asian Pacific Association for the Study of the Liver consensus recommendations on hepatocellular carcinoma. Hepatol Int 2010;4:439-474.

2. Kudo M, Okanoue T; Japan Society of Hepatology. Management of hepatocellular carcinoma in Japan: consensus-based clinical practice manual proposed by the Japan Society of Hepatology. Oncology 2007;72 Suppl 1:2-15.

3. Liu CL, Fan ST, Lo CM, Tso WK, Poon RT, Lam CM, et al. Management of spontaneous rupture of hepatocellular carcinoma: single-center experience. J Clin Oncol 2001;19:3725-3732.

4. Lai EC, Lau WY. Spontaneous rupture of hepatocellular carcino- ma: a systematic review. Arch Surg 2006;141:191-198.

5. Yang $\mathrm{Y}$, Nagano $\mathrm{H}$, Ota $\mathrm{H}$, Morimoto $\mathrm{O}$, Nakamura $\mathrm{M}$, Wada $\mathrm{H}$, et al. Patterns and clinicopathologic features of extrahepatic recurrence of hepatocellular carcinoma after curative resection. Surgery 2007;141:196-202.

6. Terada T, Takeuchi T, Hirano R, Nagata S, Kubota H, Honda S. Spontaneous rupture of peripancreatic lymph node with hepatocellular carcinoma metastasis: report of an autopsy case with massive peritoneal bleeding. Hepatol Res 2003;26:73-76.

7. Chen HW, Yang CF, Chao CC. Spontaneous rupture of peritoneal seeding hepatocellular carcinoma: report of two cases. J Acute Med 2016;6:64-66.

8. Hong SS, Kim TK, Sung KB, Kim PN, Ha HK, Kim AY, et al. Extrahepatic spread of hepatocellular carcinoma: a pictorial review. Eur Radiol 2003;13:874-882. 